Pacific Journal of Mathematic 


\title{
A CHARACTERIZATION OF GENERAL Z.P.I.-RINGS II
}

\section{KATHLEEN B. Levitz}

\begin{abstract}
A commutative ring $R$ is a general Z.P.I.-ring if each ideal of $R$ can be represented as a finite product of prime ideals. If $R$ is not a general Z.P.I.-ring, it is still possible that each principal ideal of $R$ can be represented as a finite product of prime ideals. In this paper, it is shown that if $R$ is a commutative ring in which each ideal generated by two elements can be written as a finite product of prime ideals, then $R$ must be a general Z.P.I.-ring.
\end{abstract}

Let $R$ be a commutative ring. $R$ is a general Z.P.I.-ring if each ideal of $R$ can be represented as a finite product of prime ideals. In a previous paper, we proved that $R$ is a general Z.P.I.-ring if each finitely-generated ideal of $R$ can be represented as a finite product of prime ideals [4; Theorem 2.3]. If each ideal of $R$ generated by $n$ or fewer elements can be represented as a finite product of prime ideals, then we define $R$ to be a $\pi(n)$-ring. Mori completely characterized the structure of $\pi(1)$-rings in a series of four papers $[5,6$, $7,8]$. Using his characterization, it is not difficult to construct a $\pi(1)$-ring that is not a $\pi(n)$-ring for any $n>1$. For this reason it is surprising that the main result of this paper is the following theorem.

THeOREM. Let $R$ be a commutative ring. Then the following conditions are equivalent:

(a) $R$ is a general Z.P.I.-ring;

(b) for $n \geqq 2, R$ is a $\pi(n)$-ring;

(c) $R$ is a $\pi(2)$-ring.

Throughout this paper, $R$ denotes a commutative ring and $n$ denotes an arbitrary positive integer.

2. $\pi(n)$-rings without zero-divisors. If $D$ is an integral domain, we call a prime ideal $P$ of $D$ minimal if $P$ is of height one. An integral domain $D$ with identity is a Krull domain if there is a set of rank one discrete valuation rings $\left\{V_{\alpha}\right\}$ such that $D=\bigcap_{\alpha} V_{\alpha}$ and such that each nonzero element of $D$ is a non-unit in only finitely many of the $V_{\alpha}$.

Example 2.1. An integral domain $D$ with identity is a $\pi(1)$-ring if and only if $D$ is a Krull domain in which each minimal prime ideal 
is invertible [4; Theorem 1.2]. If $Z$ denotes the rational integers, then the polynomial ring in one indeterminate $Z[x]$ is a $\pi(1)$-ring and $Z[x]$ is not a $\pi(n)$-ring for any $n>1$.

Henceforth we refer to $\pi(n)$-rings without zero-divisors as $\pi(n)$ domains.

Lemma 2.2. Let $R$ be a $\pi(2)$-domain with identity. Then $R$ is a. Krull domain in which each prime ideal of height one is invertible. Moreover, the prime ideals of height one are pairwise comaximal.

Proof. If $R$ is a $\pi(2)$-domain, $R$ is a $\pi(1)$-domain. It follows from [4; Theorem 1.2] that $R$ is a Krull domain in which each minimal prime ideal is invertible. Let $P_{1}$ and $Q$ be distinct minimal prime ideals of $R$. Let $a \in P_{1} \mid Q$. Then

$$
(a)=\prod_{i=1}^{s} P_{i}^{e_{i}}
$$

where, for each $i, e_{i} \geqq 1, P_{i} \neq Q$, and $P_{i}$ is a minimal prime ideal. Let $b \in Q \backslash \bigcup_{i=1}^{s} P_{i}$. Then

$$
(a, b)=\prod_{j=1}^{m} R_{j} ;\left(a, b^{2}\right)=\prod_{k=1}^{p} S_{k},
$$

where for each $j$ and $k, R_{j}$ and $S_{k}$ are prime ideals of $R$.

If $b t \in(a)$ for some $t \in R$, then $(b t) \subset \prod_{i=1}^{n} P_{i}^{e_{i}}$. If for each $i$, $1 \leqq i \leqq s$, we let $v_{i}$ denote the valuation on $R$ with respect to the minimal prime ideal $P_{i}$, then $v_{i}(b t) \geqq e_{i}$ while $v_{i}(b)=0$. Hence $t \in P_{i}^{\left(e_{i}\right)}$, the $e_{i}$ th symbolic power of $P_{i}$. Since for each $i, P_{i}$ is invertible, it follows that $P_{i}^{\left(e_{i}\right)}=P_{i}^{e_{i}}$ [9; Lemma 21], and so $t \in P_{i}^{e_{i}}$. Because each $P_{i}$ is invertible, we can use an induction argument on $s$ to conclude that $t \in \prod_{i=1}^{s} P_{i}^{e_{i}}=(a)$.

If $\bar{R}=R /(a)$, and $\bar{b}$ is the image of $b$ in $\bar{R}$, the above argument. shows that $\bar{b}$ is a regular element of $\bar{R}$. In $\bar{R}$,

$$
\begin{aligned}
(\bar{b}) & =\prod_{j=1}^{m}\left(R_{j} /(a)\right) \\
\left(\bar{b}^{2}\right) & =\prod_{k=1}^{p}\left(S_{k} /(a)\right) .
\end{aligned}
$$

By [1; Theorem 1], the factorization of the ideal $\left(\overline{b^{2}}\right)$ is unique up to. factors of $\bar{R}$. It follows that $p=2 m$, and that we can index the ideals $S_{k}, 1 \leqq k \leqq p$, so that

$$
R_{j}=S_{2 j-1}=S_{2 j} \text {. }
$$

Hence $\left(a, b^{2}\right)=\prod_{k=1}^{p} S_{k}=\prod_{j=1}^{m}\left(R_{j}\right)^{2}=(a, b)^{2}$. Thus 


$$
(a) \subset\left(a, b^{2}\right)=(a, b)^{2} \subset\left(a^{2}, b\right) .
$$

If $x \in(a)$, then $x=r a^{2}+s b$, where $r, s \in R$. This implies that $s b \in$ $(a)$, and, consequently, $s \in(a)$. We conclude that

$$
(a) \subseteq(a)(a, b) \text {. }
$$

Since the reverse conclusion is always valid,

$$
(a)=(a)(a, b) \text {. }
$$

Because $a \neq 0$, it follows that

$$
R=(a, b) \subseteq\left(P_{1}, Q\right) \subseteq R
$$

Hence the minimal prime ideals of $R$ are comaximal. This completes the proof of the lemma.

An integral domain with identity that is a general Z.P.I.-ring is called a Dedekind domain.

Theorem 2.3. Let $R$ be an integral domain with identity. The following conditions are equivalent:

(1) $R$ is a Dedekind domain,

(2) for $n \geqq 2, R$ is a $\pi(n)$-domain;

(3) $R$ is a $\pi(2)$-domain.

Proof. $(1 \rightarrow 2)$ By definition of Dedekind domain.

$(2 \rightarrow 3)$ By definition of $\pi(n)$-ring.

$(3 \rightarrow 1)$ By Lemma 2.1, $R$ is a Krull domain in which prime ideals of height one are invertible. To conclude that $R$ is a Dedekind domain, it suffices to show that $R$ is of Krull dimension one [3; Theorem 35.16]. Each non-unit of $R$ is contained in some minimal prime ideal. Hence, if $R$ has a unique minimal prime ideal $P, P$ is also the unique maximal ideal of $R$, and $R$ is of Krull dimension one. If $R$ has more than one minimal prime ideal, then by Lemma 2.1, all these prime ideals are comaximal. If $Q$ is any nonzero proper prime ideal of $R$, there is a minimal prime ideal $P$ such that $P \subseteq Q$ [3; Corollary 35.10]. If $P \neq Q$, there exists $b \in Q \backslash P . \quad(b)=\prod_{i=1}^{t} S_{i}$, where for each $i, S_{i}$ is a minimal prime ideal of $R$ and $S_{i} \neq P$. Since $b \in Q$, for some $i, 1 \leqq i \leqq t, S_{i} \subset Q$. But this implies that $R=$ $\left(P, S_{i}\right) \subseteq Q$. Hence $Q=P$, and $R$ is of Krull dimension one. This completes the proof of the theorem.

THEOREM 2.4. Let $R$ be a $\pi(2)$-domain without identity. Then $R$ is a general Z.P.I.-ring.

Proof. Each minimal prime ideal of $R$ is a principal ideal [8; 
Theorem 26]. If $R$ contains a unique minimal prime ideal $(p)$, then it must be the case that $R=(p)$ [8; Lemma II]. We assume that $R$ contains two distinct minimal prime ideals, $(p)$ and $(q)$. Using the same argument we did in Lemma 2.2, we can show that

$$
(p)=(p)(p, q) \text {. }
$$

Since $(p)$ is a regular ideal, it follows that $R$ must have an identity [2; Corollary 5.2]. Therefore, since $R$ has no identity, it must be the case that $R$ is the only nonzero prime ideal of itself.

Let $A$ be a nonzero ideal of $R$. Then there is a smallest positive integer $n$ such that $R^{n} \subset A \subseteq R^{n-1}$. Let $a \in A \backslash R^{n}$. Since $(a)=R^{k}$ for some $k<n$, it follows that $R^{n} \subset(a)=R^{k} \leqq A \leqq R^{n-1}$. Hence $A=R^{n-1}$. Because each ideal of $R$ is a power of $R$ it follows that $R$ is a general Z.P.I.-ring [10; Theorem 2]. This completes the proof of this theorem.

\section{Main result.}

LEMMA 3.1. Let $R$ be a $\pi(2)$-ring with identity. If $R$ is the direct sum of finitely many rings, $R=\sum_{i=1}^{k} R_{i}$, then each direct summand $R_{i}$ is also a $\pi(2)$-ring.

Proof. Let $R_{j}$ be one of the direct summands of $R$, and let $A_{j}=$ $\left(a_{1 j}, a_{2 j}\right)$ be an ideal of $R_{j}$ generated by two elements of $R_{j}$. Let $e_{i}$ denote the identity of the direct summand $R_{i}, 1 \leqq i \leqq k$. Then if $A$ is the ideal of $R$ generated by the two elements $\left(\sum_{i \neq j} e_{i}\right)+a_{1 j}$ and $\left(\sum_{i \neq j} e_{i}\right)+a_{2 j}$, then

$$
A=\prod_{r=1}^{t} P_{r}
$$

where for each $r, 1 \leqq r \leqq t, P_{r}$ is a prime ideal of $R$. Then $A_{j}=$ $A R_{j}=\left(\prod_{r=1}^{t} P_{r}\right) R_{j}=\prod_{r=1}^{t}\left(P_{r} R_{j}\right)$. Since for each $r, P_{r} R_{j}$ is a prime ideal of $R_{j}, A_{j}$ can be expressed as a finite product of prime ideals. Hence $R_{j}$ is a $\pi(2)$-ring.

A principal ideal ring $R$ with identity is called a special primary ring if $R$ contains only one prime ideal $M \neq R$ and if $M^{k}=(0)$ for some positive integer $k$.

THEOREM 3.2. Let $R$ be a commutative ring. Then the following conditions are equivalent:

(a) $R$ is a general Z.P.I.-ring;

(b) for $n \geqq 2, R$ is a $\pi(n)$-ring;

(c) $R$ is a $\pi(2)$-ring. 
Proof. It is clear that (a) implies (b) and that (b) implies (c). We now show that (c) implies (a). We consider three cases: (1) $R$ is a commutative ring with identity; (2) $R$ is a commutative ring without identity, but with zero divisors; (3) $R$ is an integral domain without identity.

If $R$ is a commutative ring with identity, then $R$ is a direct sum of $\pi(1)$-domain with identity and special primary rings by [7; Hauptsatz]. Using [10; Theorem 2], we can conclude that $R$ is a general Z.P.I.-ring if any summand $R_{i}$ of $R$ that is a domain is Dedekind. From Lemma 3.1 it follows that each summand of $R$ is a $\pi(2)$-ring. Hence if the summand $R_{i}$ is a domain, $R_{i}$ is Dedekind by Theorem 2.3. Thus a $\pi(2)$-ring with identity is a general Z.P.I.-ring.

If $R$ is a commutative ring without identity, but with zero-divisors, then $R=M$ or $R=M+K$, where $K$ is a field and $M$ is a ring without identity such that each ideal of $M$ is a power of $M$ [8; Hauptsatz 11]. $R$ is a general Z.P.I.-ring by [10; Theorem 2].

The last case is settled by Theorem 2.4.

\section{RERERENCES}

1. Robert Gilmer, On factorization into prime ideals, (to appear).

2. - Multiplicative ideal theory, Vol. I, Queens University Press, Kingston, Ontario, 1968.

3. - Multiplicative ideal theory, Vol. II, Queens University Press, Kingston, Ontario, 1968.

4. Kathleen Levitz, A characterization of general Z.P.I.-rings, (to appear in Proc. Amer. Math. Soc.)

5. Shinziro Mori, Über die Produktzerlegung der Hauptideale I, J. Sci. Hiroshima Univ. (A), 8 (1938), 7-13.

6. — Über die Produktzerlegung der Hauptideale II, J. Sci. Hiroshima Univ. (A), 9 (1939), 145-155.

7. - Über die Produktzerlegung der Hauptideale III, J. Sci. Hiroshima Univ. (A), 10 (1940), 85-94.

8. — Über die Produktzerlegung der Hauptideale IV, J. Sci. Hiroshima Univ. (A), 11 (1941), 7-14.

9. Joe Leonard Mott, Multiplication rings containing only finitely many minimal prime ideals, J. Sci. Hiroshima Univ. (A-1), 33 (1969), 73-83.

10. Craig A. Wood, On general Z.P.I.-rings, Pacific J. Math., 30 (1969), 837-846.

Received September 28, 1971 and in revised form March 7, 1972.

UNIVERSITY OF KENTUCKY 



\section{PACIFIC JOURNAL OF MATHEMATICS}

\section{EDITORS}

H. SAMELSON

Stanford University

Stanford, California 94305

C. R. HobBY

University of Washington

Seattle, Washington 98105
J. DUGUNDJI

Department of Mathematics

University of Southern California

Los Angeles, California 90007

RICHARD ARENS

University of California

Los Angeles, California 90024

\section{ASSOCIATE EDITORS}

E. F. BECKENBACH

B. H. NeumanN

F WoLF

K. YoshidA

\section{SUPPORTING INSTITUTIONS}

UNIVERSITY OF BRITISH COLUMBIA

UNIVERSITY OF SOUTHERN CALIFORNIA

CALIFORNIA INSTITUTE OF TECHNOLOGY

UNIVERSITY OF CALIFORNIA

MONTANA STATE UNIVERSITY

STANFORD UNIVERSITY

UNIVERSITY OF NEVADA

NEW MEXICO STATE UNIVERSITY

OREGON STATE UNIVERSITY

UNIVERSITY OF OREGON

OSAKA UNIVERSITY

UNIVERSITY OF TOKYO

UNIVERSITY OF UTAH

WASHINGTON STATE UNIVERSITY

UNIVERSITY OF WASHINGTON

$\stackrel{*}{*} \stackrel{*}{*} \stackrel{*}{*}{ }^{*}{ }^{*}$ MMEICAN MATHEMATICAL SOCIETY

NAVAL WEAPONS CENTER

Printed in Japan by International Academic Printing Co., Ltd., Tokyo, Japan 


\section{Pacific Journal of Mathematics}

\section{Vol. 42, No. $1 \quad$ January, 1972}

Tage Bai Andersen, On Banach space valued extensions from split faces ........

David Marion Arnold, A duality for quotient divisible abelian groups of finite

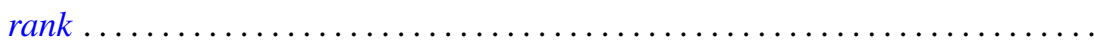

Donald Pollard Ballou, Shock sets for first order nonlinear hyperbolic

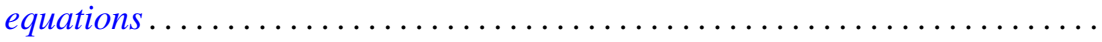

Leon Brown and Lowell J. Hansen, On the range sets of $H^{p}$ functions .........

Alexander Munro Davie and Arne Stray, Interpolation sets for analytic

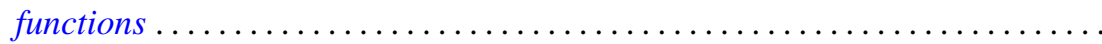

M. G. Deshpande, Structure of right subdirectly irreducible rings. II . . . . . . . . .

Barry J. Gardner, Some closure properties for torsion classes of abelian

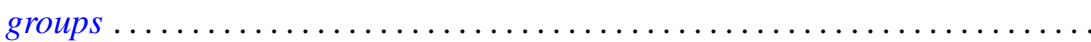

Paul Daniel Hill, Primary groups whose subgroups of smaller cardinality are

direct sums of cyclic groups . . . . . . . . . . . . . . . . . . .

Richard Allan Holzsager, When certain natural maps are equivalences .........

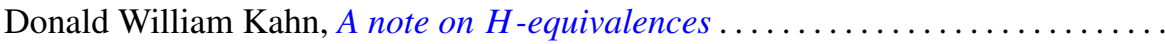

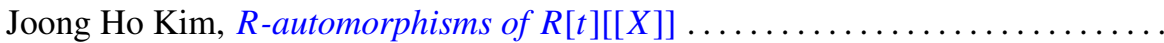

Shin'ichi Kinoshita, On elementary ideals of polyhedra in the 3-sphere.........

Andrew T. Kitchen, Watts cohomology and separability...

Vadim Komkov, A technique for the detection of oscillation of second order

ordinary differential equations .

Charles Philip Lanski and Susan Montgomery, Lie structure of prime rings of characteristic 2

Andrew Lenard, Some remarks on large Toeplitz determinants . .

Kathleen B. Levitz, A characterization of general Z.P.I.-rings. II .

Donald A. Lutz, On the reduction of rank of linear differential systems

David G. Mead, Determinantal ideals, identities, and the Wronskian ...

Arunava Mukherjea, A remark on Tonelli's theorem on integration in product

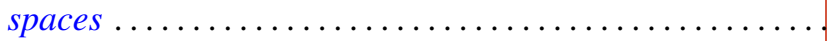

Hyo Chul Myung, A generalization of the prime radical in nonassociative rings.

John Piepenbrink, Rellich densities and an application to unconditionally nonoscillatory elliptic equations.

Michael J. Powers, Lefschetz fixed point theorems for a new class of multi-valued maps .

Aribindi Satyanarayan Rao, On the absolute matrix summability of a Fourier

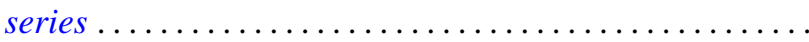

T. S. Ravisankar, On Malcev algebras ......................... 227

William Henry Ruckle, Topologies on sequences spaces . . . . . . . . . . . . . 235

Robert C. Shock, Polynomial rings over finite dimensional rings . . . . . . . . . 251

Richard Tangeman, Strong heredity in radical classes . . . . . . . . . . . . . . 259

B. R. Wenner, Finite-dimensional properties of infinite-dimensional spaces . . . . 267 